(2) Open Access Full Text Article

\title{
Lenvatinib-Induced Acute Pancreatitis in a Patient with Metastatic Thyroid Cancer: A Case Report
}

This article was published in the following Dove Press journal:

International Journal of General Medicine

\author{
Hong Jun Kim (D) \\ Jae Joon Han \\ Chi Hoon Maeng \\ Sun Kyung Baek (D)
}

Department of Internal Medicine, Kyung Hee University School of Medicine,

Seoul, Republic of Korea
Correspondence: Sun Kyung Baek

Division of Hematology-Oncology,

Department of Internal Medicine,

Kyung Hee University School of Medicine,

23, Kyungheedae-ro, Dongdaemoon-gu,

Seoul 130-872, Korea

Tel +82.2-958-8362

$\mathrm{Fax}+82.2-968-1848$

Email wkiki@naver.com
Background: Lenvatinib, a novel multi-target tyrosine kinase inhibitor, has been approved for treating differentiated thyroid cancer. Herein, we describe a rare case of acute pancreatitis that developed during lenvatinib treatment in a 65 -year-old man with recurrent thyroid cancer.

Case Presentation: The patient was admitted to our department following a complaint of acute-onset epigastric pain and indigestion. He had been receiving lenvatinib since 34 days. Although his serum amylase and lipase levels were normal, he had acute-onset persistent epigastric pain and typical computed tomography findings, which were consistent with those of acute pancreatitis. As other common etiologies were excluded, it was concluded that the patient had lenvatinib-induced acute pancreatitis. On admission day 14, he could consume food orally, after conservative care, including drug cessation, intravenous hydration, and pain control.

Conclusion: Physicians should consider acute pancreatitis as a differential diagnosis for patients complaining of abdominal pain while on lenvatinib, regardless of hyperamylasemia or hyperlipasemia. Systematic collection of data on acute pancreatitis development during lenvatinib treatment should be considered, and further research is warranted to identify the mechanism of acute pancreatitis associated with multi-target tyrosine kinase inhibitors such as lenvatinib.

Keywords: differentiated thyroid cancer, hyperlipasemia, tyrosine kinase inhibitors

\section{Introduction}

Lenvatinib is a novel multi-target tyrosine kinase inhibitor (TKI) that targets vascular endothelial growth factor receptor (VEGFR) 1-3, fibroblast growth factor receptor (FGFR) 1-4, platelet-derived growth factor receptor (PDGFR)- $\alpha$, ret protooncogene, and c-KIT. It has been approved for the treatment of differentiated thyroid cancer (DTC), ${ }^{1}$ renal cell carcinoma, ${ }^{2}$ hepatocellular carcinoma, ${ }^{3}$ and endometrial carcinoma. ${ }^{4}$ TKIs represent the only feasible treatment for DTC that is refractory to radioactive iodine (iodine-131) (RAI) therapy, ${ }^{5}$ and lenvatinib has shown considerable efficacy in the treatment of this disease. ${ }^{1,5}$ The common adverse effects of lenvatinib therapy include hypertension, peripheral edema, increased thyroid stimulating hormone level, thrombocytopenia, fatigue, anorexia, nausea, and diarrhea. As lenvatinib has recently been introduced in clinical practice, physicians should consider the possibility of its unexpected and significant complications.

Herein, we describe a rare case of acute pancreatitis that developed during lenvatinib treatment in a 65 -year-old patient with recurrent DTC. 


\section{Case Presentation}

A 65-year-old man was admitted to our department with a complaint of acute-onset epigastric pain and indigestion. He had been diagnosed with follicular thyroid cancer and received a total thyroidectomy 28 years ago. There are no medical records left, including the cancer stage at the time, but judging from the statement that the patient did not receive any treatment after surgery, it is assumed that it was early stage thyroid cancer. Twenty years after surgery, thyroid cancer recurred in the lungs, hilar lymph node, and pleura, and he underwent left lung metastasectomy, followed by three consecutive RAI treatments, from 2010 to 2012. After 5 years of observation, in December 2017, he developed symptoms such as frequent cough and chest pain, caused by aggravated lung metastases (Figure 1A), for which he started receiving $24 \mathrm{mg}$ of lenvatinib per day. After 2 weeks of TKI treatment, he developed adverse effects such as grade 2 constipation, grade 3 anorexia, grade 3 mucositis, and grade 2 myalgia; thus, the dose was reduced to $20 \mathrm{mg}$ per day. Twenty days after dose reduction, the patient presented to the emergency room of Kyung Hee University Hospital.

On admission, he complained of acute onset of persistent epigastric pain and indigestion, but had no fever, dyspnea, or diarrhea. On presentation, he had a temperature of $36.4^{\circ} \mathrm{C}$, heart rate of 83 beats per minute, respiratory rate of 20 breaths per minute, and blood pressure of 130/70 mm Hg. The patient had mild abdominal distension with hypoactive bowel sounds, and mid-epigastric tenderness to palpitation was observed during physical examination. Laboratory tests showed the following: serum total bilirubin, $0.45 \mathrm{mg} / \mathrm{dL}$ (normal, $0.3-1.2 \mathrm{mg} / \mathrm{dL}$ ); alanine aminotransferase (ALT), $50 \mathrm{IU} / \mathrm{L}$ (normal, $<50 \mathrm{U} / \mathrm{L}$ ); aspartate aminotransferase (AST), $57 \mathrm{IU} / \mathrm{L}$ (normal, $<50$ $\mathrm{U} / \mathrm{L}$ ); amylase, $22 \mathrm{U} / \mathrm{L}$ (normal, 28-100 U/L); lipase, $25 \mathrm{U} / \mathrm{L}$ (normal, $8-58 \mathrm{U} / \mathrm{L}$ ); triglyceride, $102 \mathrm{mg} / \mathrm{dL}$ (nor$\mathrm{mal},<150 \mathrm{mg} / \mathrm{dL}$ ); calcium, $7.8 \mathrm{mg} / \mathrm{dL}$ (normal, 8.8 10.6 $\mathrm{mg} / \mathrm{dL}$ ); and C-reactive protein, $15.11 \mathrm{mg} / \mathrm{dL}$ (normal, $<0.5 \mathrm{mg} / \mathrm{dL}$ ). A computed tomography scan with contrast enhanced presented heterogeneous fluid collection around and fat infiltration into the tail of the pancreas, in addition to splenic subcapsular fluid collection and secondary colitis at the descending colon, without any biliary abnormalities (Figure 2). However, it showed that thyroid cancer burden was mildly improved (Figure 1B). Although elevated serum amylase and lipase levels were not detected, acute onset of persistent epigastric pain and characteristic findings on computed tomography scan were consistent with acute pancreatitis. ${ }^{6}$ Given the absence of organ failure and systemic complications, the patient was classified as mild acute pancreatitis in accordance with the revised Atlanta classification. ${ }^{6}$

He had no recent history of alcohol consumption and a survey of his current medication list revealed no other remedy capable of inducing acute pancreatitis. Given the sequence of lenvatinib treatment and development of acute pancreatitis without any other usual sources, it was concluded that the patient had lenvatinib-induced acute pancreatitis.

The patient was conservatively treated with lenvatinib cessation, intravenous hydration, and pain control. On admission day 14, he could consume oral food without acute pancreatitis recurrence or aggravation and was later discharged. The patient refused to continue lenvatinib treatment and has only been monitoring symptoms without additional chemotherapy. In June 2020, more than 2 years later, the last imaging follow-up showed relatively indolent thyroid cancer disease course (Figure 1C).

\section{Discussion}

To the best of our knowledge, this is the first formal case report of lenvatinib-induced acute pancreatitis in a patient with metastatic, RAI treatment-refractory, thyroid cancer. Although lenvatinib-induced pancreatitis has been briefly stated in a letter to the editor ${ }^{7}$ and a case series, ${ }^{5}$ the present report will be meaningful because there has been no detailed description of the initial symptoms, diagnostic process, and subsequent clinical course of lenvatinibinduced acute pancreatitis.

DTC accounts for approximately $95 \%$ of thyroid cancers, and it is usually known to have a good prognosis. ${ }^{8}$ Surgery is the primary treatment strategy for patients with DTC, and if indicated, it is followed by RAI and thyroid hormone suppression therapies. ${ }^{9}$ However, in metastatic or recurrent cancers, treatment options could be limited. Although cytotoxic chemotherapeutic agents, such as doxorubicin, have been shown to be ineffective, several molecular pathways, including VEGFR, FGFR, PDGFR, NRAS, HRAS, BRAF, and RET/PTC, have been elucidated to be involved in DTC ${ }^{10-14}$ pathogenesis. This has led to the investigation of the use of multi-target TKIs, such as lenvatinib and sorafenib, for RAI refractory DTC treatment, for which Phase 3 trials were conducted and US Food and Drug Administration approval was obtained in 2015 and 2013, respectively. ${ }^{5,9,15}$ 

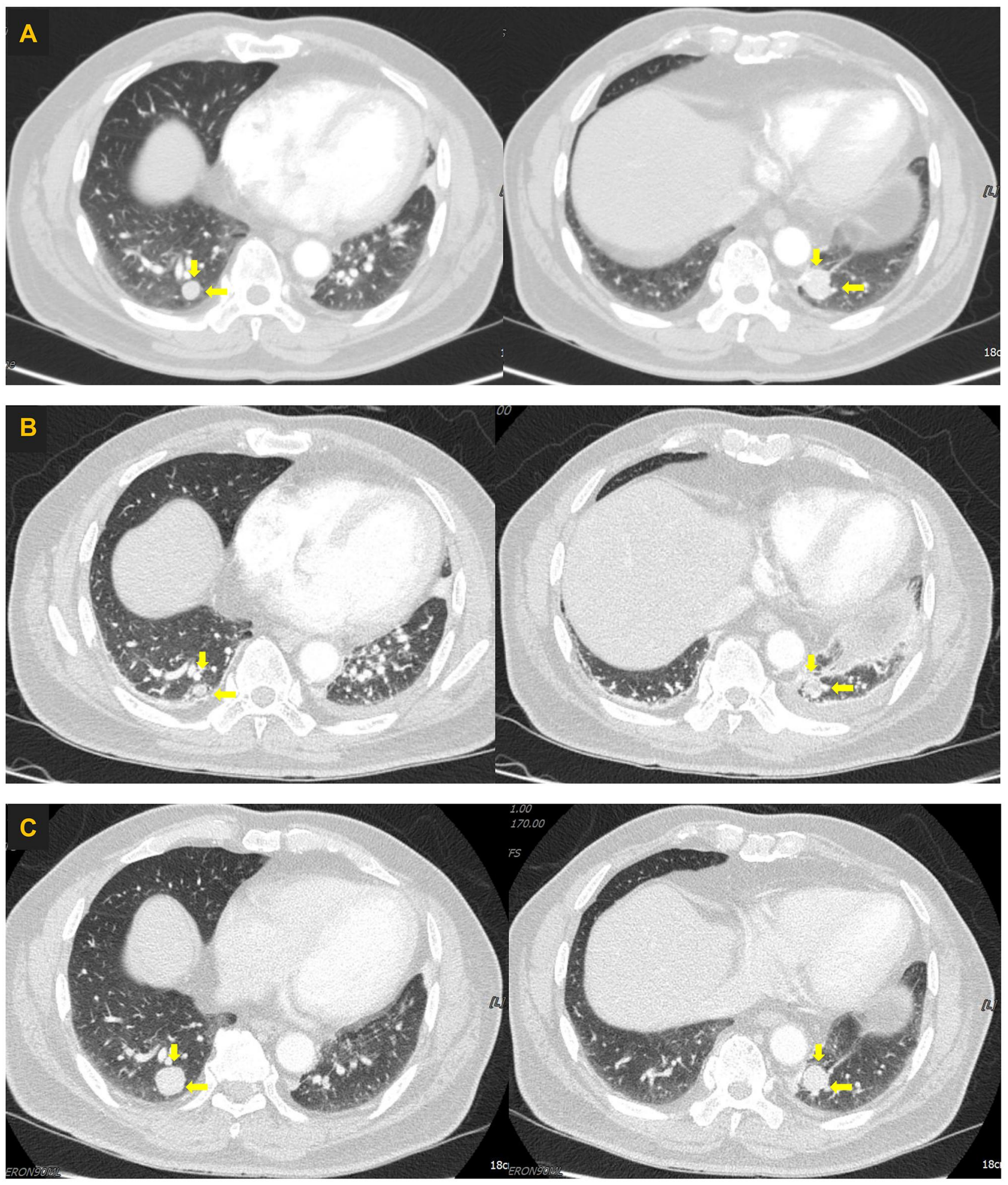

Figure I Computed tomography images of the lungs. (A) Computed tomography scan obtained in December 2017 showing increased size of metastatic nodules (yellow arrows) in both right and left lower lobes. (B) Computed tomography scan obtained in January 2018 showing slightly decreased size of metastatic nodules (yellow arrows) in both the right and left lower lobes. (C) Computed tomography scan obtained in July 2020 showing slightly aggravated metastatic nodules (yellow arrows) in both the right and left lower lobes.

Acute pancreatitis is a sudden inflammatory disorder of the pancreas. Its diagnosis requires the presence of at least two of the following three factors: sudden onset severe epigastric pain, elevated serum amylase or lipase, and typical computed tomography magnetic resonance imaging $^{6}$ scan findings. Biliary etiologies and alcohol 

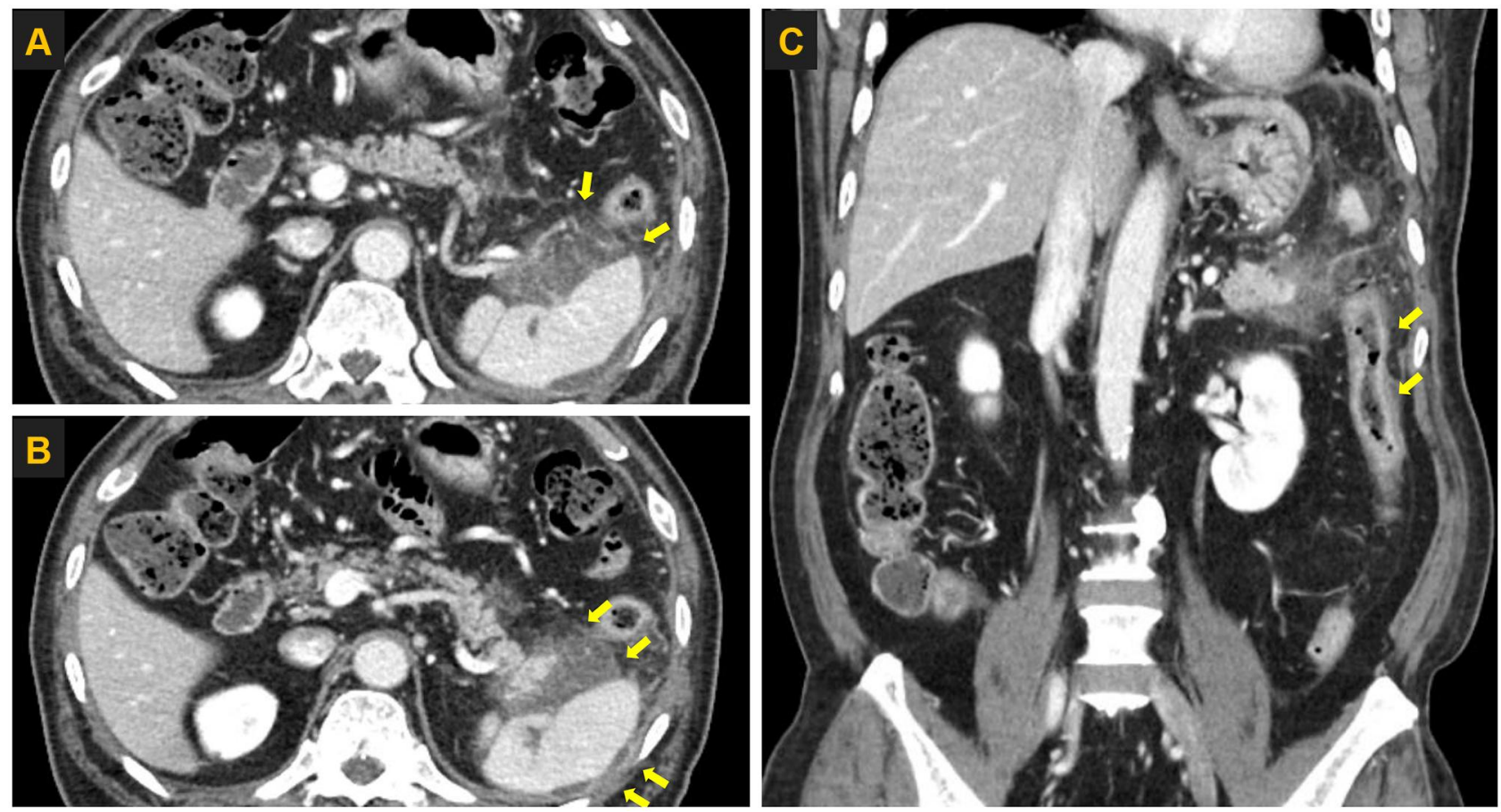

Figure 2 Computed tomography images of the abdomen obtained in January 2018. (A) Computed tomography image of the pancreas showing heterogeneous fluid collection and fat infiltration (yellow arrows) around the tail. (B) Computed tomography image showing splenic subcapsular fluid collection (yellow arrows). (C) Computed tomography image showing secondary colitis at the descending colon (yellow arrows).

abuse are the main causes of acute pancreatitis, accounting for approximately $60 \%$ to $75 \%$ of the cases, while hypertriglyceridemia, hypercalcemia, and infections are less common causes. ${ }^{16}$ Drugs such as aminosalicylates, sulfonamides, diuretics, and corticosteroids, are also considered a relatively rare cause of acute pancreatitis, accounting for approximately $0.1 \%$ to $2 \%$ of all cases. ${ }^{17}$ Because druginduced pancreatitis has no specific clinical features, an exclusion diagnosis is needed, ${ }^{18}$ as with the current case, after biliary abnormalities, alcohol abuse history, and other drug intoxication were excluded.

Although data on lenvatinib-induced acute pancreatitis is scarce, a recent systematic review has shown that the association between all-grade pancreatitis and other multitarget TKIs, including sunitinib, sorafenib, pazopanib, vandetanib, and axitinib, is statistically significant. ${ }^{19}$ However, the pathogenesis of multi-target TKI-induced acute pancreatitis is unclear. Multi-target TKIs are known to increase serum amylase or lipase levels; ${ }^{20}$ however, the correlation between hyperlipasemia/hyperamylasemia and TKI-induced acute pancreatitis remains unclear. One possible mechanism by which TKIs result in acute pancreatitis is pancreatic duct obstruction via reflux of duodenal contents resulting from decreased gastrointestinal motility. ${ }^{21}$ Another explanation is that VEGF inhibition results in acinar cell apoptosis and provocation of autodigestive enzymes. However, given that monoclonal antibodies targeting VEGF are not associated with pancreatitis, these events could be off-target effects of VEGFR TKIs. ${ }^{19}$

\section{Conclusion}

In conclusion, the current case suggests that physicians should consider acute pancreatitis as a differential diagnosis in patients who complain of abdominal pain while receiving lenvatinib, regardless of hyperamylasemia or hyperlipasemia. More prudent monitoring is required in patients at a higher risk of pancreatitis, with biliary abnormalities or with a history of alcoholism. Moreover, for those with several comorbidities, lenvatinib administration should be reconsidered carefully as its benefits might not outweigh its risks. Systematic collection of data on the development of acute pancreatitis during lenvatinib treatment should be considered, and further research is warranted to identify the mechanism underlying multi-target TKI-induced acute pancreatitis.

\section{Abbreviations}

DTC, differentiated thyroid cancer; TKI, tyrosine kinase inhibitor; VEGFR, vascular endothelial growth factor receptor; FGFR, fibroblast growth factor receptor; 
PDGFR, platelet-derived growth factor receptor; RAI, radioactive iodine (iodine-131).

\section{Ethics and Consent Statement}

Based on the regulation of the department of research of the Kyung Hee University of medical science, institutional review board approval is not required for case reports.

Written informed consent was obtained from the patient for publication of this case report and any accompanying images.

\section{Author Contributions}

All authors made substantial contributions to conception and design, acquisition of data, or analysis and interpretation of data; took part in drafting the article or revising it critically for important intellectual content; agreed on the journal to which the article will be submitted; gave final approval of the version to be published; and agree to be accountable for all aspects of the work.

\section{Funding}

There is no funding to report.

\section{Disclosure}

The authors have no conflicts of interest to declare for this work.

\section{References}

1. Schlumberger M, Tahara M, Wirth LJ, et al. Lenvatinib versus placebo in radioiodine-refractory thyroid cancer. $N$ Engl $J$ Med. 2015;372 (7):621-630. doi:10.1056/NEJMoa1406470

2. Motzer RJ, Hutson TE, Ren M, Dutcus C, Larkin J. Independent assessment of lenvatinib plus everolimus in patients with metastatic renal cell carcinoma. Lancet Oncol. 2016;17(1):e4-5. doi:10.1016/ s1470-2045(15)00543-4

3. Kudo M, Finn RS, Qin S, et al. Lenvatinib versus sorafenib in first-line treatment of patients with unresectable hepatocellular carcinoma: a randomised phase 3 non-inferiority trial. Lancet. 2018;391 (10126):1163-1173. doi:10.1016/s0140-6736(18)30207-1

4. Makker V, Rasco D, Vogelzang NJ, et al. Lenvatinib plus pembrolizumab in patients with advanced endometrial cancer: an interim analysis of a multicentre, open-label, single-arm, phase 2 trial. Lancet Oncol. 2019;20(5):711-718. doi:10.1016/s1470-2045(19)30020-8

5. Balmelli C, Railic N, Siano M, et al. Lenvatinib in advanced radioiodine-refractory thyroid cancer - a retrospective analysis of the swiss lenvatinib named patient program. $J$ Cancer. 2018;9(2):250-255. doi: $10.7150 /$ jca. 22318
6. Banks PA, Bollen TL, Dervenis C, et al. Classification of acute pancreatitis-2012: revision of the Atlanta classification and definitions by international consensus. Gut. 2013;62(1):102-111. doi:10.1136/gutjnl-2012-302779

7. Kawakami H, Kubota Y, Ban T, Shibata N, Hosokawa A. Lenvatinibinduced acute pancreatitis associated with a pancreatic pseudocyst and splenic pseudoaneurysms. Pancreas. 2018;47(6):e34-e35. doi:10.1097/mpa.0000000000001061

8. Hundahl SA, Cady B, Cunningham MP; U.S. and German Thyroid Cancer Study Group, et al. Initial results from a prospective cohort study of 5583 cases of thyroid carcinoma treated in the united states during 1996: an American college of surgeons commission on cancer patient care evaluation study. Cancer. 2000;89(1):202-217.

9. Haugen BR, Alexander EK, Bible KC, et al. 2015 American thyroid association management guidelines for adult patients with thyroid nodules and differentiated thyroid cancer: the American thyroid association guidelines task force on thyroid nodules and differentiated thyroid cancer. Thyroid. 2016;26(1):1-133. doi:10.1089/thy.2015.0020

10. Salajegheh A, Smith RA, Kasem K, et al. Single nucleotide polymorphisms and mRNA expression of VEGF-A in papillary thyroid carcinoma: potential markers for aggressive phenotypes. Eur J Surg Oncol. 2011;37(1):93-99. doi:10.1016/j.ejso.2010.10.010

11. St Bernard R, Zheng L, Liu W, Winer D, Asa SL, Ezzat S. Fibroblast growth factor receptors as molecular targets in thyroid carcinoma. Endocrinology. 2005;146(3):1145-1153. doi:10.1210/en.2004-1134

12. Nikiforov YE. RET/PTC rearrangement in thyroid tumors. Endocr Pathol. 2002;13(1):3-16. doi:10.1385/ep:13:1:03

13. He G, Zhao B, Zhang X, Gong R. Prognostic value of the BRAF V600E mutation in papillary thyroid carcinoma. Oncol Lett. 2014;7 (2):439-443. doi:10.3892/ol.2013.1713

14. Volante M, Rapa I, Gandhi M, et al. RAS mutations are the predominant molecular alteration in poorly differentiated thyroid carcinomas and bear prognostic impact. J Clin Endocrinol Metab. 2009;94 (12):4735-4741. doi:10.1210/jc.2009-1233

15. Brose MS, Nutting CM, Jarzab B, et al. Sorafenib in radioactive iodine-refractory, locally advanced or metastatic differentiated thyroid cancer: a randomised, double-blind, phase 3 trial. Lancet. 2014;384(9940):319-328. doi:10.1016/s0140-6736(14)60421-9

16. Lankisch PG, Apte M, Banks PA. Acute pancreatitis. Lancet. 2015;386(9988):85-96. doi:10.1016/s0140-6736(14)60649-8

17. Shelton C, LaRusch J, Whitcomb DC, et al. Pancreatitis overview. In: Adam MP, Ardinger HH, Pagon RA, editors. Genereviews(®). Seattle: University of Washington, Seattle Copyright (C) 1993-2020, University of Washington, Seattle. GeneReviews is a registered trademark of the University of Washington, Seattle. All rights reserved; 1993.

18. Spanier BW, Tuynman HA, van der Hulst RW, Dijkgraaf MG, Bruno MJ. Acute pancreatitis and concomitant use of pancreatitis-associated drugs. Am J Gastroenterol. 2011;106(12):2183-2188. doi:10.1038/ ajg.2011.303

19. Ghatalia P, Morgan CJ, Choueiri TK, Rocha P, Naik G, Sonpavde G. Pancreatitis with vascular endothelial growth factor receptor tyrosine kinase inhibitors. Crit Rev Oncol Hematol. 2015;94(1):136-145. doi:10.1016/j.critrevonc.2014.11.008

20. Pezzilli R, Corinaldesi R, Morselli-Labate AM. Tyrosine kinase inhibitors and acute pancreatitis. Jop. 2010;11(3):291-293.

21. Li M, Srinivas S. Acute pancreatitis associated with sorafenib. South Med J. 2007;100(9):909-911. 


\section{Publish your work in this journal}

The International Journal of General Medicine is an international, peer-reviewed open-access journal that focuses on general and internal medicine, pathogenesis, epidemiology, diagnosis, monitoring and treatment protocols. The journal is characterized by the rapid reporting of reviews, original research and clinical studies across all disease areas. The manuscript management system is completely online and includes a very quick and fair peer-review system, which is all easy to use. Visit http://www.dovepress.com/ testimonials.php to read real quotes from published authors. 